\title{
Status of the D0 Fiber Tracker and Preshower Detectors
}

\author{
Dmitri Smirnov for the D0 Collaboration
}

\begin{abstract}
In this report we focus on the performance of the D0 central fiber tracker and preshower detectors during the high luminosity $p \bar{p}$ collisions at $\sqrt{s}=1.96 \mathrm{GeV}$ delivered by the Tevatron collider at Fermilab (Run IIb). Both fiber tracker and preshower detectors utilize a similar readout system based on high quantum efficiency solid state photo-detectors capable of converting light into electrical signals. We also give a brief description of the D0 detector and the central track trigger, and conclude with a summary on the central tracker performance.
\end{abstract}

Key words: D0 scintillating fiber tracker, preshower detectors, visible light photon counters, track trigger, D0 AFEII-t electronics PACS: 07.77.Ka, 29.40.Mc, 85.60.Gz

\section{The Tevatron and the Do Detector}

The Tevatron located at Fermi National Accelerator Laboratory is a superconducting synchrotron capable of accelerating proton $(p)$ and anti-proton $(\bar{p})$ beams in opposite directions to energies of $980 \mathrm{GeV}$. Particles in the beams are grouped in 36 spatially separated bunches which are brought into the head-on collisions at the center of the D0 detector [1,2] at $396 \mathrm{~ns}$ intervals.

As a typical collider detector, the D0 detector has a tracking system, electromagnetic and hadronic calorimeters, and muon chambers to measure the energy, momentum, and charge of particles produced in the collisions. The central tracking system consists of a silicon microstrip tracker (SMT) and a central fiber tracker (CFT) both located within a $2 \mathrm{~T}$ superconducting solenoidal magnet, with designs optimized for tracking and vertexing at pseudorapidities $|\eta|<3$ and $|\eta|<2.0$ respectively. The central and forward preshower detectors (CPS and FPS) positioned just outside of the superconducting coil are used to improve the measurement of energies of electron and photon showers detected by the D0 calorimeter system. A liquid-argon and uranium calorimeter has a central section (CC) covering pseudorapidities $|\eta|$ up to $\approx 1.1$, and two end calorimeters (EC) that extend coverage to $|\eta| \approx 4.2$, with all three housed in separate cryostats. A muon system, at $|\eta|<3$, consists of layers of tracking detectors and scintillation trigger counters surrounding $1.8 \mathrm{~T}$ toroid magnets [3].

In Run IIb, with initial instantaneous luminosities reaching over $3 \times 10^{32} \mathrm{~cm}^{-2} \mathrm{~s}^{-1}$ and more than $5 p \bar{p}$ interactions per bunch crossing, the D0 detector faces stringent chal- lenges. In the following sections, we will discuss the CFT and PS detectors, and the central track trigger (CTT) hardware, as well as upgrades performed on the readout electronics aimed to improve identification of charged particles.

\section{The Central Fiber Tracker}

A schematic cross sectional view of one quarter of the CFT is shown in Fig. 1. It consists of eight thin coaxial carbon-fiber cylinders, each supporting two layers of overlapping scintillating fibers (doublets), each fiber having a $0.835 \mathrm{~mm}$ diameter. Tracking in the CFT is done by detecting the scintillation light produced when ionizing particles traverse the fibers. A small fraction of generated light is trapped inside and propagates along the fiber [4]. In the CFT, the light is read out from one end while the other end of the fiber is mirrored. In order to decrease the losses of light due to escaped photons, each fiber has 2 clads with different refraction indices. Each doublet layer is $\approx 0.28 \%$ of radiation length (c.f. to the D0 solenoid which is about $\sim 1$ radiation length). The inner doublet layer on each cylinder has fibers oriented parallel to the beam axis whereas fibers in the outer doublet layer are oriented at $+3^{\circ}$ or $-3^{\circ}$ stereo angle relative to the axis. The radii of the innermost and the outermost cylinders are $20 \mathrm{~cm}$ and $52 \mathrm{~cm}$ respectively. The number of fibers per cylinder increases with its radius, resulting in a total of $76,800 \mathrm{CFT}$ channels. The length of the CFT cylinders is $252 \mathrm{~cm}$, except for the inner two, which have length of $166 \mathrm{~cm}$. Position resolution of fiber doublets is on the order of $100 \mu \mathrm{m}$, while location of individual fibers in $r-\phi$ view is known to an accuracy of better 


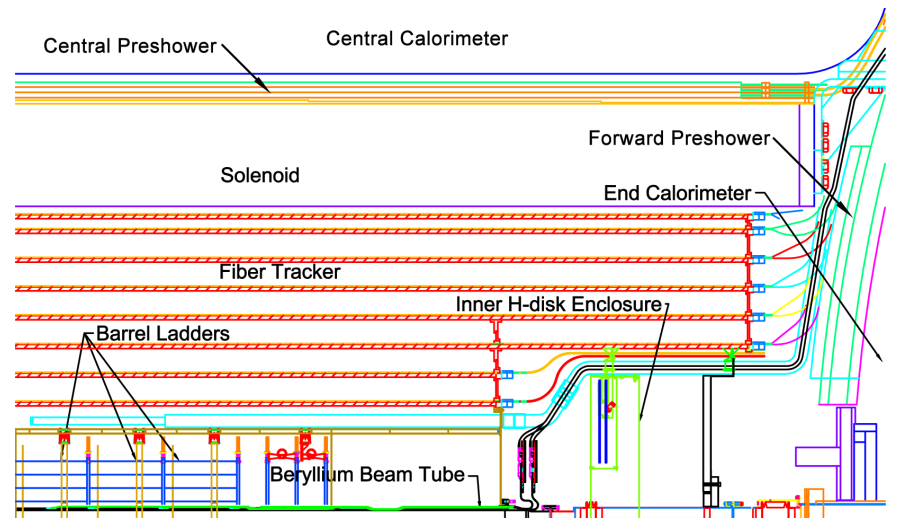

Fig. 1. Cross sectional view of one quarter of the central part of the D0 detector. The CFT is submerged in a uniform (within 0.5\%) $2 \mathrm{~T}$ magnetic field created by a $2.8 \mathrm{~m}$ long superconducting solenoidal magnet with a mean radius of $60 \mathrm{~cm}$. The CPS surrounds the solenoid, while the FPS is mounted in front of the end calorimeter.

than $50 \mu \mathrm{m}$. We detect approximately 10 photons from a minimum ionizing particle crossing a fiber (averaged over all fibers and $\eta$ ).

Each scintillating fiber is mated, via a connector, to a clear fiber waveguide. The length of waveguides ranges from $8.2 \mathrm{~m}$ to $11.4 \mathrm{~m}$ with attenuation length of about $8 \mathrm{me}-$ ters. The light signal is transferred to a solid state photodetector, or Visible Light Photon Counter (VLPC) [5].

\section{The Preshower Detectors}

The CPS and FPS detectors [6] are located just outside of the superconducting coil in front of the calorimeters as shown in Fig. 1. Both detectors are made of scintillator strips with co-axially embedded wavelength-shifting fibers. Similar to the CFT, the light signal from each strip is propagated to individual VLPC detectors via 8 to $14 \mathrm{~m}$-long waveguides.

The CPS has a cylindrical shape covering the $|\eta|<1.3$ region and is mounted in the $51 \mathrm{~mm}$ gap between the solenoid and the central calorimeter cryostat at a radius of $71 \mathrm{~cm}$. The detector is composed of three concentric cylindrical layers of $\approx 6 \mathrm{~mm}$ wide triangular plastic scintillator strips. The triangular shape strips, arranged in an interlocked base-up-base-down pattern, have been chosen for an advantage over other geometrical shapes in order to improve position resolution. Strips in the inner layer are oriented axially while those in the middle and outer layers form $\pm 22.5^{\circ}$ stereo angles with the axis. The total number of CPS channels is 7,680.

The FPS covers the forward and backward regions within a pseudorapidity range of $1.5<|\eta|<2.5$. The detector consist of two halves mounted on the heads of the end calorimeter cryostat (Fig. 1). Each half has two MIP layers immediately followed by a lead-steel absorber, whose purpose is to initiate electron and photon showers. The absorber is succeeded by two shower layers sampling energy in the sprays of particles. Each active layer in the FPS is made out of 8 evenly spaced trapezoidal wedges in total covering slightly more than $\frac{1}{2}$ of the full $2 \pi \phi$-coverage. The next successive in $z$ layer is clocked by $22.5^{\circ}$ to complete the full $\phi$ coverage. The FPS layers are composed of two sublayers of triangular scintillating strips roughly perpendicular to the beam axis and oriented at $22.5^{\circ}$ stereo angles w.r.t. each other. The total number of FPS channels is 14,968 .

The primary function of the CPS detector is to distinguish electromagnetic showers from hadronic ones and to augment the calorimeter energy measurement which would otherwise be degraded due to the material in the solenoid coil. Both the CPS and FPS can also be used for triggering on electrons, which allows the experiment to suppress the output rate of major electron backgrounds such as pion decays.

\section{The VLPC and Readout Electronic}

The VLPC is a solid-state detector with high gain and very high quantum efficiency $(\mathrm{QE})$, sensitive to light in the visible frequency range. These devices operate at a temperature of about $9^{\circ} \mathrm{K}$ and a bias in the range of 6 to $8 \mathrm{~V}$. The VLPC is easily able to distinguish single photons and has a very low gain dispersion, as shown in Fig. 3a. The detectors are manufactured in wafers, diced into 176 chips with eight $1 \mathrm{~mm}$ pixels arranged in $2 \times 4$ arrays. Test bench studies have shown that the gain of the VLPC chips can vary from 20,000 to 60,000 electrons per detected photon. The $\mathrm{QE}$ also varies among the chips with the typical values measured to be $\sim 80 \%$. The chips were sorted and grouped with the higher gain chips assigned to read out the CFT axial fibers. More details on VLPC properties can be found in $[7]$.

In D0, VLPCs are grouped into cassettes, with 1024 VLPC pixels per cassettes. The lower portion of the cassette is immersed in Helium gas at about $6^{\circ} \mathrm{K}$, while the upper portion supports a pair of analog front-end boards (AFE), on either side of the cassette, which are responsible for proper control and readout of the VLPCs. A total of 99 cassettes are used to instrument the fiber tracker and the preshower detectors. Each cassette is composed of 8 sections (modules), each serving 128 channels.

As the only electronics interfacing to VLPC chips, the AFE controls the stability of the VLPC temperature and bias voltages on per module basis. The temperature variations are controlled with a precision of much better than $\pm 0.05 \mathrm{~K}$ using a system of two carbon resistors with one serving as a heater and the other as a temperature sensor. The electronics can control the bias voltage to $\pm 30 \mathrm{mV}$. In addition, the front-end electronics is responsible for the following. It amplifies the tiny VLPC signals, pipelines them for later readout, and, on demand, digitizes them to 8 bits. Also every $396 \mathrm{~ns}$, or bunch crossing, the AFE sends a discriminator bit for every channel above some threshold ${ }^{1}$ to

1 The thresholds for the CFT channels are determined in a calibration run such that only $1 \%$ of noise exceeds the threshold, while the 
the trigger system. Based on discriminated CFT hits, the trigger system is able to make a preliminary decision on the number and momentum of the tracks. This information is then used in the trigger decision.

As part of the Run IIb upgrade, all VLPC-based detectors have been instrumented with a new version of the AFE board, AFEII-t. This board extends the capabilities of the D0 detector at very high luminosities and also provides a new capability of measuring the timing of the hits. This capability can be used to determine the rough location of the hits along the scintillating fibers, which is hoped to improve track reconstruction at high occupancies encountered at the highest instantaneous luminosities.

For the preshower detectors, gains of AFE boards are reduced to deal with significantly larger signals produced by electromagnetic showers.

\section{The Central Track Trigger}

There are three consecutive trigger levels at D0. Each level applies a set of increasingly strict requirements on the detector data in order to identify and select potentially interesting events. The Level 1 trigger is implemented in custom designed hardware and firmware with the intention to provide quick pattern recognition of electron, muon, and jet candidates. Level 2 decisions are based on a combination of the first level objects with some additional information from other subdetectors. The final level in the trigger chain consists of software algorithms whose purpose is to select events based on a simple particle reconstruction and overall event quality.

The CTT [8] is an essential part of the D0 Level 1 trigger. The CTT uses discriminated signals from the axial CFT layers to form track candidates in the $r-\phi$ plane (Fig. 2). The fiber hits are compared to predefined charged particle paths in 4 different $P_{T}$ bins with thresholds of $1.5 \mathrm{GeV}$, $3 \mathrm{GeV}, 5 \mathrm{GeV}$, and $10 \mathrm{GeV}$. If a CFT track candidate is identified, an attempt is made to match it to the preshower data. The result of this combination is used to flag electron candidates accordingly. The final list of CTT tracks is also used by the Level 1 muon and calorimeter trigger systems. This facilitates improvement of the quality of muon and electron candidates as well as forming $\tau$ candidates by using available calorimeter clusters. Furthermore, on the second trigger level, the CTT track candidates are used to seed the track finding algorithm which searches hits from the silicon microstrip tracker.

In Run IIb, the CTT has undergone a significant upgrade in order to cope with increasing instantaneous luminosities. The original hardware limited the number of CFT hit combinations to about 16,000 per $4.5^{\circ}$ sector in $\phi$ thus forcing the trigger to consider only two-fiber hits in each axial doublet layer. Such artificially reduced CFT granularity lead to very high trigger rates at high luminosities.

$\overline{\text { threshold }}$ for PS channels is 3 MIPs.

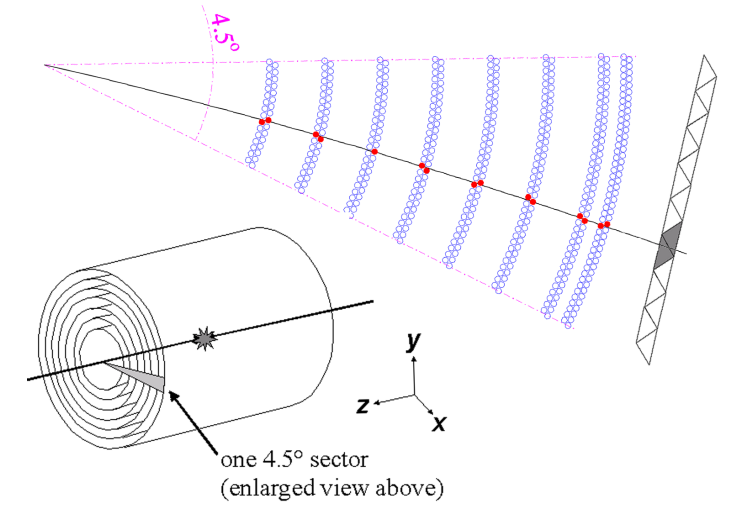

Fig. 2. Schematic view of the 8 axial layers of the CFT with an end view of one of eighty $4.5^{\circ}$ sectors enlarged. A charged particle produces hits in the scintillating fibers surrounding the interaction region. The CTT finds particle tracks and matches them to energy clusters in the PS detectors.

The upgraded CTT employs new chips capable of storing about 50,000 equations per sector. That allows use of single fiber hits to form tracks, while keeping the same latency for track finding. In addition, the CTT now also provides a hit-count based veto against high occupancy events.

\section{Performance}

Since its installation in 2000 and commissioning in 2001 the CFT and PS detectors have been in stable operation. The initial fraction of unresponsive channels in the CFT was $\approx 0.3 \%$, and only after complications due to a power outage in 2003 the number of such channels increased to $\approx 2 \%$ improving slowly with time to the present level of $\approx 1.7 \%$. We believe the unresponsive channels are due to loss of mechanical contact between VLPC pixels and readout electronics. About $0.1 \%$ of the VLPC chips have "hot" pixels that cannot distinguish individual photons. The gain of VLPCs can be measured by injecting a small amount of light into scintillating fibers with an LED system mounted on the CFT barrels. We find that the VLPC gains remain unchanged since installation.

As of April 2007, all VLPC-based detectors are fully instrumented with the AFEII-t boards. The new electronics is easier to maintain, provides more uniform pedestals and individual thresholds, and requires only infrequent calibrations.

One of the basic characteristics associated with CFT performance is the probability of measuring a signal above threshold when a fiber is truly hit by a particle. To estimate this efficiency, we reconstruct tracks in a special way by skipping all hit clusters in one doublet layer at a time. Among all tracks we select those having 15 hits in all other layers and, in addition, we apply tight requirements on track's quality in order to decrease chances of selecting tracks made of random combination of hits. Then each track is projected to the skipped layer. If the nearest track cluster resides within $\sigma \phi_{\mathrm{Dxx}}$, where $\sigma \phi_{\mathrm{Dxx}}$ is the fit uncertainty on the track's $\phi$ at Dxx doublet (this amounts to 


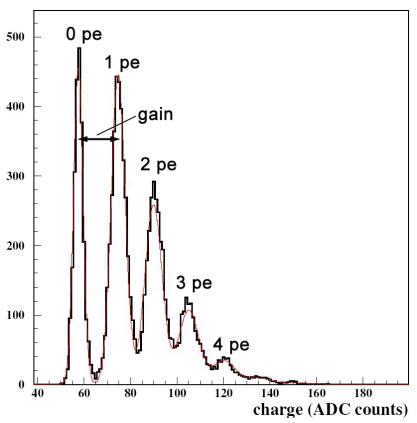

(a)

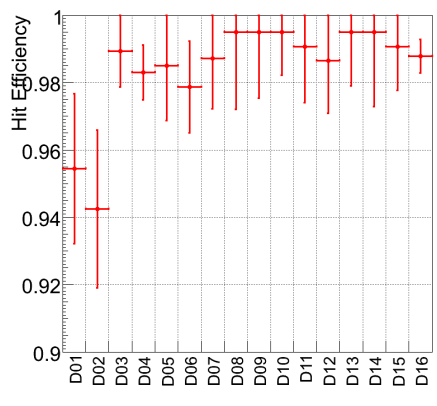

(b)
Fig. 3. (a) Charge distribution from a VLPC pixel with the peaks corresponding to $0,1,2, \ldots$ converted photons (signal induced via LED pulser). (b) Average single hit efficiency for each of the 16 doublet layers. Unresponsive channels are excluded from this assessment.

$\sim 2-5 \mathrm{~mm}$ ) around the track projection, it is assumed that this cluster would have been associated with the track in the standard reconstruction. The ratio of tracks with all 16 hits to all selected tracks gives the single hit efficiency shown in Fig. 3b for all CFT doublets, for data collected at luminosities in the range 305 to $60 \times 10^{30} \mathrm{~cm}^{-2} \mathrm{~s}^{-1}$. Unresponsive channels are not included in the calculation.

An added feature of the AFEII-t board is its capability of providing information about the time of arrival of signal generated by a charged particle in the fiber. The correlation between the hit position and the arrival time is expected to improve rejection of fake tracks. We observe good agreement between the measured speed of light and the expected one in the fiber (Fig. 4). Time resolution is estimated to be 3 ns for signals greater than 5 photo-electrons. This implies a position resolution of hits along a fiber of about $50 \mathrm{~cm}$.

The CPS performance was significantly improved by enhanced dynamic range via modified electronic gain. As the result of this upgrade, the dynamic range increased from 13 to 54 MIPs while the measured energy remains in a good agreement with the corresponding measurement in the calorimeter (Fig. 5). There is an ongoing effort to implement a similar improvement for the FPS detector.

Combined, the silicon and scintillating fiber trackers provide a precise measurement of impact parameter of tracks relative to the interaction location. On average the impact parameter resolution for high $P_{t}(>10 \mathrm{GeV})$ tracks reaches values of $20 \mu \mathrm{m}$, whereas in the special case of single high $P_{t}$ muons the impact parameter can be determined with a precision close to $\approx 10 \mu \mathrm{m}$. This allows a significant measurement of the secondary vertex from a $B$-meson decay. The efficiency of tagging a jet as one coming from a $b$ quark depends on the jet's energy. The maximum efficiency of $\approx 55 \%$ is reached in jets with energies between 40 and $100 \mathrm{GeV}$. For light-quark jets, the probability of misidentification of their origin to be a $b$ quark normally does not exceeds $3 \%$.
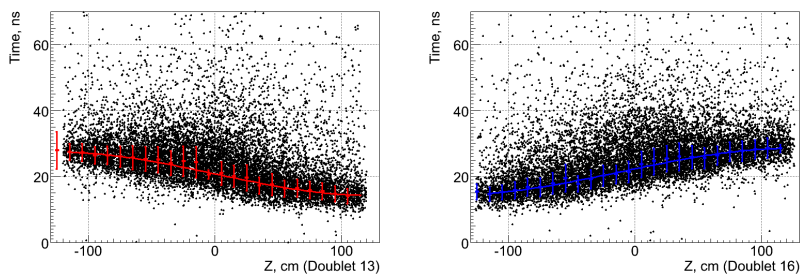

Fig. 4. Scatter plots show correlation between the $z$ position of the hit and the time of hit arrival as measured with AFEII-t in the axial (left) and stereo (right) layers. Note that stereo and axial layers are read out from opposite ends.

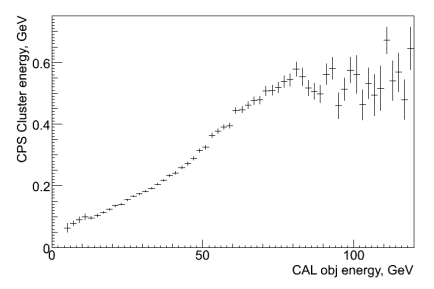

Fig. 5. The energy deposited by a shower in the CPS is well correlated with the measurement in the calorimeter over the entire active range.

\section{Conclusions}

The upgrade of the D0 detector for Run IIb was designed to explore a wide range of interesting problems in high energy physics at increasing peak luminosities. Many physics analyzes depend on tracker performance, thus it is essential for the experiment to use the full potential offered by the VLPC-based front-end electronics.

With the new CFT and PS readout electronics there is an opportunity for D0 to further improve track finding. In particular, incorporation of the hit arrival time should help the tracking at luminosities over $3 \times 10^{32} \mathrm{~cm}^{-2} \mathrm{~s}^{-1}$. Overall the D0 CFT and PS detectors are in a good shape and have very stable performance.

\section{References}

[1] S. Abachi et al. [D0 Collaboration], "The D0 Detector," Nucl. Instrum. Meth. A 338, 185 (1994).

[2] V. M. Abazov et al. [D0 Collaboration], "The upgraded D0 detector," Nucl. Instrum. Meth. A 565, 463 (2006).

[3] V. M. Abazov et al. [D0 Collaboration], "The muon system of the Run II D0 detector," Nucl. Instrum. Meth. A 552, 372 (2005).

[4] R. Ruchti, "Tracking with scintillating fibers," Nucl. Phys. Proc. Suppl. 44, 308 (1995).

[5] M. G. Stapelbroek and M. D. Petroff, "Visible light photon counters for scintillating fiber applications. II: Principles of operation," Workshop on Scintillating Fiber Detectors (SCIFI 93), Notre Dame, IN, 24-28 Oct 1993, World Scientific (1995) 621-629.

[6] P. Baringer et al. [D0 Collaboration], "Cosmic ray tests of the D0 preshower detector," Nucl. Instrum. Meth. A 469, 295 (2001).

[7] A. Bross, E. Flattum, D. Lincoln, S. Grünendahl, J. Warchol, M. Wayne and P. Padley, "Characterization and performance of visible light photon counters (VLPCs) for the upgraded D0 detector at the Fermilab Tevatron," Nucl. Instrum. Meth. A 477, 172 (2002).

[8] J. Anderson et al. [D0CTT group], "The D0 central track trigger," IEEE Trans. Nucl. Sci. 51, 345 (2004). 